\title{
A Review of Stress analysis of Helical Compression Spring for Toggle Switch Mechanism
}

\author{
Sayli. H. Bhagwat ${ }^{1}$ and S. K. Dahake ${ }^{2}$ \\ Research Scholar ${ }^{1}$ and Professor ${ }^{2}$ \\ Department of Mechanical Engineering \\ MET'S Institute of Engineering \\ Pune University, Nashik, Savitribai \\ India
}

\begin{abstract}
The country India is good for becoming a supplier of the goods and services. Where a power supply system is the main ingredient for a country's economic growth and quality of life. Nowadays installed transmission capacity for evacuation is an emerging bottleneck, distribution systems are outdated having huge losses are the cause of concern, there is a need to upgrade transmission and distribution infrastructure. The switchgear industry continues to innovate and upgrade its products to meet the evolving/future needs of its customers. From the works of literature, we are identified to analyze the mechanism of the switchgear, where most of the failure takes place due to guiding of helical compression spring in the mechanism. Hence we need to avoid this failure by analysis of spring using analytical, FEA and experimental method. FEA analysis takes place by modeling of spring using PRO-E 4.0 and Meshing and analysis can be done by Ansys 14.5 Software. Experimental stress analysis of spring done by using the universal testing machine with the help of strain gauge mounting on the spring. Results of Analytical and FEA validate with Experimental results.
\end{abstract}

Key Words: PRO-E, Ansys, Spring, Strain Gauge, Stress.

\section{INTRODUCTION}

In an electric power system, switchgear is the combinations of electrical and mechanical switches, fuses or circuit breakers. It is used to control, protect and isolate electrical equipment. Switchgear is used both to de-energize equipment to allow work to be done and to clear faults downstream. The earth switch mainly consists of fixed contacts and moving contacts. In normal "ON" condition of earth switch, these two contacts are physically connected with each other due to applied mechanical pressure on the moving contacts. The fixed contacts of earth switch carry large fault power. Due to large power high arcing produced in contact during operation of earth switch. To avoid or minimize the effect of arcing travelling distance of moving contact and velocity of moving contact are the important parameter.

To avoid or minimize the effect of arcing in medium voltage earth switch, velocity of moving contact must be high with proper sliding \& clamping profile. This project report provides the design and analysis of mechanism which generates discontinuous motion of moving contact and vanishes the arcing between contacts during operation of earth switch. Material with special consideration shall be mandatory for high temperature application. After design \& simulation of earth switch for specified requirement, it should withstand in experimental analysis. Experimental analysis will be conducted in international laboratory like CESI Italy, KEMA Korea and CPRI India.

The work presented in this paper focuses on design and analysis of earth switch for higher system rating in the range of $25 \mathrm{kA}$ to $40 \mathrm{kA}$. The specification of earth switch should be realistic to meet the functional requirements for the given application. Over-specification results in costly and bulky design without much value addition. The ideas explored here will be helpful to 
Sayli. H. Bhagwat et. al., Stress analysis of Helical Compression Spring for Toggle Switch ....

practicing engineer to specify correct parameters of earth switch. This will result in procuring the most economical earth switch without sacrificing end user requirements.

\subsection{Problem Statement}

- Design and Development of Helical compression spring for Toggle Switch mechanism which is used for $40 \mathrm{kA}$ current rating in Switch gear industry. Need to define proper kinematic mechanism for analysis and synthesis of mechanism. Force and Velocity analysis for the mechanism in order to find the maximum force and velocity developed.

- In addition to this, it is very crucial to have optimized design of spring for toggle switch mechanism, which will provide required velocity.

\section{OBJECIVE}

- Prediction of Maximum and Minimum force developed during working condition of Mechanism.

- Finding out the stresses and deformation on helical compression Spring.

- Application of Regression analysis for predicting the relation between different parameters.

\section{LITRATRE REVIEW}

Manan Deb et al. (2014) has given the general information about the toggle switching. Toggle mechanisms are used in electrical switches. This paper defines and classifies the toggle phenomena observed during switching. The concept of double toggle introduced in this paper enables a systematic screening of kinematic structure for the suitability in high performance switches. Seven structural and three kinematic criteria are identified for this purpose. It is also demonstrated that each such feasible kinematic structure lends itself to multiple physical embodiments. Therefore, the theory and procedure presented in this work can be used for design of numerous kinematic distinct mechanisms. An electric switch can be as simple as a knife switch where a conducting pivoted lever is directly manipulated using an insulating knob by the operator to make or break a circuit. The fixed contact is typically Ushaped which also acts as a clip to hold the lever in position when the circuit is closed. In a switch there is a link for user input, called the handle which moves a current carrying member (follower) to make a specific region on it called moving contact, come in contact with another specific region on a fixed link called fixed contact. These contacts are made of special geometry and material suitable for good electrical performance under repeated impacts during making and breaking of the circuits. Early developments indicate use of an external spring to maintain the position of the handle in one of the two states, ON and OFF. The moving contact here was mounted on an elastic strip (beam) which was deformed by the actuation lever to establish contact. The switch uses the same type of contacts, but the elasticity of the elastic strip itself is used to maintain the ON and OFF states through the cam profile used in the operating lever. Although this is named as a toggle switch and it mentions about quick closing and breaking of the circuit, within the context of the patent, its effectiveness is not very clear. The use of heavy duty, dedicated, actuation springs can be found in different applications. However, the fixed contacts used here are U-shaped as in knife switches. The spring is said to work over the 'deadcentre' configuration. Mechanism enabling such phenomenon is referred to as bi-stable, snap-action and toggle mechanisms in literature. In this paper we preferred to use the term 'toggle'.

The theory developed here is applied in the design of low voltage circuit breaker mechanism. The present paper focuses on the structural synthesis for the switching mechanisms using the strategy of function assignment. It emphasizes the importance of the toggle phenomenon, introduces the notion of double toggle in multi degree of freedom mechanisms and shows how such mechanisms can be designed for different switching patterns. The paper introduces the notion of active and passive sub-chains to systematically derive the options for the function assignment and uses them for developing the set of structural and kinematic conditions to be satisfied by a mechanism to exhibit double toggle. The working of the operating mechanism of a few commonly available switches has been critically studied from the perspective of toggling to evaluate the designs. The theory developed is then used for the design of a new double toggle type switching mechanism. The paper is relevant for all manually operated toggle switches. More specifically, the paper purely focuses on the switching mechanism of the circuit breaker. The tripping mechanism and other fault sensing techniques are potential future studies and out of the scope of the present paper. An optimization method to satisfy the stringent demand on performance for a specific switching application is also not in the scope.

Manan Deb et al. (2014) has given the general information about a special multi-degree of freedom toggle behaviour called double toggle. The paper identified and characterized a special multi-degree of freedom toggle behaviour, called double toggle, observed in a typical MCCB switching mechanism. For an idealized system, the condition of toggle sequence is derived geometrically. The existing tools available in a multi-body dynamics package are used for exploring the dynamic behaviour of such systems parametrically. The double toggle mechanism is found to make the system insensitive to the operator's behaviour; however, the system is vulnerable under extreme usage. The linkage kinematics and stopper locations are found to have dominant role on the behaviour of the system. It is revealed that the operating time is immune to the inertial property of the input link and sensitive to that of the output link. Novel designs exploiting this observation, in terms of spring and toggle placements, to enhance switching performance have also been reported in the paper. Detailed study revealed that strategic placement of the spring helps in selective 
alteration of system performance. Thus, the study establishes the critical importance of the kinematic design of MCCB over the dynamic parameters.

Mahani Yusoff et al. (2013) have given the general information about Effect of Sintering Parameters on Microstructure and Properties of Mechanically Alloyed Copper- Tungsten Carbide Composite. In this study, copper-tungsten carbide composite was produced by mechanical alloying and powder metallurgy. The effect sintering temperature and time $\left(800^{\circ} \mathrm{C}\right.$ to $1000^{\circ} \mathrm{C}$ and 1 to $\left.3 \mathrm{~h}\right)$ on composite microstructure and properties were investigated. The results showed that WC is formed at lower temperature and the presence of WC favoured the enhancement of the properties of the composite. The increased both hardness and electrical conductivity has been observed with the increase sintering temperature. Copper-based composite benefits from its excellent electrical properties, thermal conductivity and good resistance to oxidation are mostly utilized in electrical application. Copper-based composite is as a promising material for electrical contact which is widely used in high voltage switches, circuit breakers, relays and welding electrodes. Carbide is frequently used as reinforcement material that is homogenously dispersed in copper matrix. The advantage is they can retard particle coarsening during annealing, in order to retain the strength of the composite. Tungsten carbide is a refractory metal with a high melting point $\left(2777^{\circ} \mathrm{C}\right)$, high hardness $(1000 \mathrm{~kg} / \mathrm{mm} 2)$ at elevated temperatures, excellent resistance to oxidation and a high thermal stability. Copper-tungsten carbide composite electrical contact has been fabricated mostly by powder metallurgy through powder blending, compacting and sintering. Powder metallurgy able to disperse very fine reinforced particles into copper, leading to a better control of microstructure.

Stobrawa and Rdzawski have shown that fine separated tungsten carbide particles are embedded in copper matrix with the enhancement of composite density and hardness. On the other hand, powder metallurgy coupled with mechanical alloying imparts a large amount of interest among several researchers have shown that fine separated tungsten carbide particles are embedded in copper matrix with the enhancement of composite density and hardness. On the other hand, powder metallurgy coupled with mechanical alloying imparts a large amount of interest among several researchers is enhances by sintering in order to produce density controlled materials by applying thermal energy. Solid state sintering occurs when the green compact is densified entirely in a solid state at particular sintering temperature. Many factors can be addressed to contribute composite sintering abilities. They include green density, constituent element, particle size, heating rate, sintering temperature, sintering time and sintering environment. Mechanical alloying mechanism is used to promote the diffusion process by producing Nano structured while sintering is expected to enhance the diffusion by forming a new phase. Solid state sintering for copper-based composite usually occurs at sintering temperature of 800 to $1000^{\circ} \mathrm{C}$. Therefore, in the present work, the effects of sintering parameters on copper-tungsten carbide composite prepared by powder metallurgy and mechanical alloying were investigated. The properties of sintered composite were also discussed.

Predrag Bojic et al. (2013) addresses the study of a high-speed earthing switch in gas-insulated metal enclosed switchgear. A high-speed earthing switch (HSES) is a permanently installed switching apparatus in gas-insulated metal enclosed switchgear (GIS). A spring drive driven HSES has been developed and tested in one-phase and three-phase synthetic test circuit. The HSES rated voltages of $245 \mathrm{kV}$ and $300 \mathrm{kV}$, enable to perform closing (making) operation on the peak value of short circuit current of 20 $\mathrm{kA}$ without needing maintenance. This paper presents the main task of an HSES at application in GIS, design characteristics, and two different test procedures. Wide searching throughout standards was made in order to define the real status of this apparatus. Earthing switches (work-in-progress earthing switches or busbar earthing switches, for example) are used for properly connecting deenergized live parts of the high voltage system to the grounding system. On the outgoing side of the feeders, a make-proof version (high-speed) is frequently used to dissipate inductive and capacitive currents from parallel cables or overhead lines or to reduce the risk to the GIS system in case of faulty connections. In the insulated design they are also used for measuring purposes and for testing protection relays. In the switchgear type up to $245 \mathrm{kV}$, the earthing switches are of a pin-type design. Depending on the switchgear design, they are either incorporated in a common housing with the disconnection switches or installed in a separate housing. With the pin-type earthing switch, the earthing pin at earth potential is pushed into the matching contact. Make-proof earthing switches are equipped with a stored-energy spring mechanism. The spring, which stores the required switching energy, can be recharged either with a motor or manually in an emergency.

Nadezda Talijan (2011) addresses the study on Electrical contact materials based on silver. Composite electrical contact materials based on silver and oxides of different metals have been used for many years in different types of electrical devices for making and breaking of electrical circuit. $\mathrm{Ag}-\mathrm{CdO}$ materials have been most commonly used over the years as the materials for electrical contacts and other electrical components due to their excellent electrical and mechanical properties, mainly for small and medium-current loads. Recent investigations have led to the development and implementation of new environmental friendly electrical contact materials in which the toxic $\mathrm{CdO}$ is replaced by less harmful metal oxides, while maintaining the good final properties. It was found that the $\mathrm{Ag}-\mathrm{SnO} 2$ composite material meets most of the required properties. Results of experimental 
Sayli. H. Bhagwat et. al., Stress analysis of Helical Compression Spring for Toggle Switch ....

investigations of different technological procedures for obtaining various types of silver based electrical contact materials are presented in this paper through defined technological phases with optimized process parameters.

Rong-Fong Fung et al. (2011) addresses the study motion of control of a toggle mechanism actuated by an electro hydraulic system. This control system is non-linear and time varying one due to the internal leakage of electro hydraulic system as well as the non-linear dynamic of toggle mechanism. The specified position-velocity profile of the output slider of the toggle mechanism is considered as the desired trajectory. Bing

Ravikant et al. (2013) has given the general information about the objective of the drive shaft. This drive shaft is connected with the transmission shaft with the help of universal joint. Here finite element analysis of a drive shaft has been taken as a case study. The modal analysis of a drive shaft has been carried out the inherent frequencies and vibration mode shapes with their respective deformation. The maximum stress point and dangerous areas are found by the deformation analysis of drive shaft. The relationship between the frequency and the vibration modal is explained by the modal analysis of drive shaft.

J.Anjaneyulu et al. (2013) has given the general information about Analysis and Vibration Behaviour of a Medium Voltage Spring Operated Switch Gear Mechanism used for Circuit Breaker. In recent years, considerable attention has been given to the analysis of flexible mechanisms. For mechanisms which operate at high speeds, the inertial forces become so large and they cannot be ignored and the mechanism members undergo considerable deformation. Under these situations the rigid body assumption is no longer valid and hence the need arises for consideration of the flexibilities of the members of the mechanism. This paper investigates dynamic response of a spring operated mechanism of a $69 \mathrm{kV}$ SF6 (Sulphur hexa-Fluoride) circuit breaker during its opening operation. In the present work, a spring operated mechanism is analysed using the multi body simulation. The kinematic synthesis of the mechanism considering its links to be rigid is derived. In kinematic analysis the velocity and acceleration of each link is calculated and their responses are plotted with the time during opening operation of the mechanism. This method is particularly important in analysing the dynamics of complicated mechanisms. The dynamic response of the circuit breaker with different spring constants was studied and its effect on the breaking time was also presented. The switchgear mechanism's link dimensions are verified by performing the mechanism motion simulation by using software. The proto type model of the switch gear mechanism is fabricated and tested for studying the dynamic response of the links in the mechanism for its operation. Automation has led to higher operating speeds and also high accuracy requirement by the industry led to use more sophisticated tools. The assumption of rigid members however is not justified always, in particular for the modern machinery operating at high speed, where due to its inertia effect a linkage may experience a significant deformation which is adequate to disturb the performance of its intended function and actual operation.

G.E. Agafonov et al. (2013) have given the general information about High Speed Grounding Switch for Extra High Voltage lines. At a single-phase short-circuit in extra-high voltage lines after breaking of faulty phase from both sides by the circuit breakers the secondary arc can exist for a long time in a place of a short-circuit, roused by electrostatic induction from the "sound" phases. One of the possible ways of a secondary arc elimination is employment of grounding switches, which are capable during a dead time at auto reclosing of circuit breakers at first to short-circuit purposely a faulty phase from both sides, thus having shunted and eliminated an arc in a fault location, and then to open it, by breaking of the induced currents, and to make a phase ready for making of the circuit breakers, i.e. renewal of power supply. The basic requirements to a switching performance of grounding switch result from the transient analysis in power lines and include the requirements to switching of capacitive and inductive currents, including currents, which up to $100 \mathrm{~ms}$ have no zero value. At a single-phase short circuit in extra-high voltage lines the circuitbreakers usually break a short circuit current on both sides of faulted phase. After breaking the short circuit current a secondary arc flows in a place of short-circuit, moved by electrostatic induction from the "sound" phases. In this case if the interaction between phases is great, the secondary arc will not be quenched during a dead time before the circuit-breakers' reclosing. One of the ways of secondary arc elimination is the use of high speed grounding switches (HSGS), which arrange the condition for extinguishing of a secondary arc by grounding of a faulted phase from both sides after short circuit arc quenching. After quenching of a secondary arc HSGS' disconnect the induced currents: the first in time HSGS disconnects an electromagnetic induced current, and the second electrostatic current. After the breaking operation the conditions are arranged for the circuit-breakers' making on the ends of the faulted phase. HSGS should perform the making and breaking operations during a dead time. The number of the particular requirements keeps remote the high speed grounding switches with respect to other switchgear. In particular, among such requirements are relatively small interrupted currents at very large TRV value. Generally, for SF6 HSGS the design should be singlebreak, i.e. to provide an arc quenching and full insulation to earth of a system by one break. The conditions can be arranged in a line, when the induced electromagnetic current switched by HSGS, will have no zero value within up to $80 \mathrm{~ms}$, i.e. the maximum arcing time, given by environment, should make $80 \mathrm{~ms}$. On the basis of these requirements the HSGS design was developed and manufactured. The basic elements of HSGS design are an arcing device, reducer, drive and metal construction.

Kelgandre Kiran et al. (2011) have given the general information about Development of 40kA Earthing Switch by Using Industrial Product Design Model. The main function of earthing switch is to earth the residual current inside the switchboard cabling and current carrying parts of copper and aluminium. It is used to earth the high voltage current coming through the source and flow 
through the switchgear. There are different types and versions of earthing switch like high voltage, low voltage indoor, outdoor earth switch. Several versions of Earthing Switches for different applications like Maintenance Earthing Switches. These are single pole or three pole units manually operating mechanism with a provision of filling motor mechanism. High Speed Earthing Switches are operated by spring energy. Spring is charged by motor mechanism. Earthing switch has ratings like 6kA, 12kA, 20kA, 25kA, 31.5kA etc. Earthing Switch is necessary to earth the conducting parts before maintenance and also to provide deliberate short-current while testing. There can be three types of earthing switches in metal-clad Switches; manually operated, automatic high speed Earthing Switch, protective Earthing Switch for earthing the installation. Special high speed Earthing Switches with interrupting capability are also available.

The earthing switch has a snap action closing mechanism which functions independently of the rotation of the drive shaft. The switching speed and torque achieved in this process are independent of the actions of the operating mechanism In the opening process, in contrast, the compression springs have no effect on the speed of contact separation or suitable manual or motorized operating mechanism with the necessary torque for the type of switch and an operating angle 90 can be fitted for operation of the switch. Earthing switches with fault making capacity can be reliably closed against short circuit currents, thus protecting the operator and switchgear in the cause of inadvertent operation. Naturally, operation behind a closed compartment door forms part of the overall cubicle safety. Every earthing switch is fitted with a spring operating device which makes the closing capacity independent of the operating speed. The control side of the earthing switch and direction of current flow can usually be chosen without limitations except for a few models with integral current transformers. Materials used in manufacturing the earth switch parts which is important are the frame is zinc plated steel plate, the current carrying parts are silver plated copper and the insulators are made of cast epoxy resin. Earthing switches do not need maintenance in normal service. They can withstand 2000 operations without load and two operations under the full short current rating. The contacts can be changed if the closed position contact surface is badly worn. A reasonable amount of switching arc burning around the knife edge or contact tips will not affect the closing capacity of the earthing switches. The contacts have been lubricated with special paraffin which does not normally need to be renewed. If required the contact surfaces can be greased with lubricating stick. Insulator support requires for the mounting of earth switch in the panel with proper sustaining capability which is made up of epoxy resin. Type tests are mandatory as per IEC standards. This test are carried out to prove short-circuit making performance of earthing switches, satisfactory operation at temperature limit and proper function of position indicating devices.

\section{METHODOLOGY}

For the development of high speed earthing switch, we have followed industrial product design theories. Topic discussed here is the study that product design and development is a set of activity in which ideas and needs are given a physical form initially as solution concept and then as a specific configuration. Product Design \& Development is the set of activity beginning with the perception of market opportunity and ending with production sale and delivery of product. Fig 1.4 shows the engineering design is the centralized activity.

$\begin{array}{ll}\text { - } & \text { Selecting the spring design. } \\ \text { - } & \text { Literature Review } \\ \text { - } & \text { Numerical analysis. } \\ \text { - } & \text { Spring modeling in Pro-E. } \\ \text { - } & \text { Experimental analysis. } \\ & \text { Result analysis and validation. }\end{array}$

\section{CONCLUSION}

- In this way we are doing prediction of Maximum and Minimum force developed during working condition of Mechanism.

- $\quad$ Also finding out the stresses and deformation on helical compression Spring.

- Validation of results of Analytical, FEA with experimental analysis after completion of work.

\section{REFERENCE}

[1] Manan Deb, DibakarSen, "Design of double toggle switching mechanisms", Mechanism and Machine Theory, Volume71, 2014,Pp-163-190.

[2] Manan Deb, DibakarSen, "Parametric study of the behaviour of double toggle switching mechanism", Mechanism and Machine Theory, Volume 63, 2013, Pp- 8-27.

[3] Rong-Fong Fung, Rong-Tai Yang, "Motion Control of an Electrohydraulic Actuated Toggle Mechanism", Mechatronics, Volume 11, 2011, Pp-939-946. 
Sayli. H. Bhagwat et. al., Stress analysis of Helical Compression Spring for Toggle Switch ....

[4] Bing Bai, Lixiang Zhang, Tao Guo, Chaoqun Liu, "Analysis of Dynamic Characteristics of the Main Shaft System in a Hydro- $\quad$ turbine Based on ANSYS", volume 31, 2012, Pp- 654 - 658.

[5] Ravikant, GopalKrishan, MukeshDidwania, "Modal analysis of drive shaft using FEA", Volume-3, 2013, Pp-4-7.

[6] Clive Maxfield, "Electrical Engineering",Newnes is an imprint of Elsevier, 2008.

[7] Ming,M.,Jeom, K.,"Ultimate strength based stiffened panel design using multi-objective optimization method and its application to ship structure" Proc. of the 12th International Symposium, Korea,2013(25),pp.1-5.

[8] Gaol,X., et.al." Dimension Optimization of Through Silicon Via(TSV) through Simulation and Design of Experiment (DOE)" 2012 International Conference on Electronic Packaging Technology \& High Density Packaging, 2012,11(12)pp.1185-1189.

[9] Charkha,P.G., Jaju,S.B., “Analysis \& Optimization of Connecting Rod” ICETET 2009,pp.86-91. 